\title{
Espoir banlieues, plan B
}

\section{Bertrand Cassaigne}

DANS ReVUe PROJET 2009/2 ( ${ }^{\circ} 309$ ), PAGES 84 À 87

ÉDITIONS C.E.R.A.S

\section{ISSN $0033-0884$}

DOI 10.3917/pro.309.0084 
spoir banlieues, plan B

Bertrand Cassaigne

L

ambition est d'ouvrir de nouvelles voies. Reconnaître la gravité de la fracture qui se creuse dans les quartiers en difficulté, au risque parfois d'une ghettoïsation, prendre acte de l'échec des politiques de la ville précédentes, proposer une autre démarche, au autre "Plan ", porteur d'espoir pour les banlieues, telle était l'ambition de Fadela Amara et du président Nicolas Sarkozy. Un an après, cette ambition se heurte à bien des résistances et suscite bien des scepticismes. S'il est sans doute un peu tôt pour tirer un premier bilan exhaustif, on peut cependant s'interroger sur ses débuts - comme l'a fait, le 20 janvier, le Comité interministériel des villes.

\section{Deux ambitions}

Ce plan apparait comme le croisement de deux "philosophies ", parfois convergentes mais parfois divergentes, dans leurs diagnostics et leurs propositions. Fadela Amara, venue du terrain, du monde associatif, entend rompre avec les cloisonnements des politiques publiques. Le plan se veut résolument "partenarial " et "interministériel ». Elle a tenu avant son élaboration à rencontrer pendant six mois des responsables d'associations, des élus, des citoyens. Et c'est bien la concrétisation de leurs espoirs, de leurs projets, qu'elle proposait de réaliser. Elle parle de rupture avec des "pratiques venues d'en haut ", avec "l'empilement des dispositifs ", avec une méthode stigmatisante - une "logique d'exception " - en " mobilisant les administrations de droit commun ».

Pour Nicolas Sarkozy, la politique de la ville s'était trop réduite jusque là à une politique d'assistance - les ministères se défaussant de la question des banlieues pour en faire un problème social, qui ne relèverait de leur responsabilité que sous la forme d'une contribution financière. A ses yeux, surtout, elle était pensée en direction des lieux, des territoires, plus que des personnes elles-mêmes. Or ce sont d'abord les gens qu'il faut mettre en mouvement pour leur permettre de sortir du repli, de la séparation. 
Concrètement, ces deux ambitions se traduisent par des démarches difficiles à concilier. Toutes deux se rejoignent pour refuser d'identifier la réponse avec une politique d'assistance, qui requiert toujours plus de financements sans s'attaquer au mal à la racine. Toutes deux s'inscrivent dans une logique du résultat, avec des dispositifs contractuels, des programmes évaluables, des objectifs donnés sur trois ans pour chaque ministère concerné... Mais quand l'une appelle une mobilisation collective - rétablir des liens en soutenant les associations, en veillant à la présence de services publics et de commerces, en s'appuyant sur les entreprises -, l'autre pense d'abord à une mobilisation individuelle ("le ghetto n'est pas seulement dans les murs, il est dans les têtes ") soutenue par un système de sanctions et de récompenses - une politique de sécurité renforcée, un accès à "l'égalité des chances" promis aux plus méritants ${ }^{1}$.

\section{Des priorités}

En pratique, les différents volets de ce $4^{\mathrm{e}}$ plan Banlieue ne diffèrent pas fondamentalement des programmes antérieurs, mais des accents nouveaux se veulent porteurs d'espoir ${ }^{2}$. Le premier traduit la volonté de faire de l'emploi le vecteur essentiel de l'intégration. Un contrat d'autonomie est proposé aux plus mobilisés des jeunes de moins de 26 ans. Alors que beaucoup trop dépendent de dispositifs d'aide sociale, un accompagnement sur un an (six mois avant la signature du contrat, six mois après) doit leur permettre de trouver, en passant par une entreprise d'insertion ou par une formation qualifiante, une place dans le monde du travail. Le contrat est signé entre le jeune et un organisme rémunéré en fonction des résultats. Le chiffre de 100000 contrats en trois ans est avancé, mais un premier objectif de 4500 fixé pour début 2008 ne sera atteint que ce trimestre ! En revanche, l'engagement national des grandes entreprises à recruter des jeunes des quartiers semble avoir été davantage tenu : 11500 d'entre eux ont obtenu, en 2008, un CDI ou un CDD de plus de six mois. D'autres mesures visent à soutenir les porteurs de projet : la création de 20000 entreprises est prévue, grâce à un guichet unique des dispositifs d'aide et à des prêts d'honneur de la Caisse des Dépôts, etc. L'ambition est aussi que la fonction publique reflète davantage localement la diversité de la population (par l'octroi de bourses, de préparation aux concours). Pour autant,

1. Cf. Jacques Donzelot, Esprit, mars-avril 2008.

2. Cf. une présentation détaillée par Julien Damon, Regards sur l'actualité, La Documentation française, juillet 2008 . 
un plan emploi de véritable ampleur serait nécessaire, quand on sait que la crise économique vient désormais bousculer en premier les habitants des banlieues : intérimaires, stagiaires, CDD seront les premiers touchés.

Une deuxième priorité est l'éducation, considérée comme assurant les conditions de la réussite pour tous. Elle prévoit de généraliser l'accompagnement éducatif dans toutes les Zep (primaire et collège), en accueillant les élèves pendant deux heures après les cours. Mais cette mesure compensera-t-elle la décision prise par ailleurs d'une réduction des postes "Rased ", en direction des enfants qui ont le plus de difficultés ? Par ailleurs, bien des associations de soutien scolaire ne seront plus aidées. Le volet éducation annonce aussi le renforcement des écoles de la deuxième chance (12000 places annoncées), même si leur coût reste une limite - on attend le concours des entreprises! Parallèlement, ce sont 30 pôles d'excellence qui doivent être créés dans les quartiers sur tout le territoire, des sections internationales, des filières artistiques... L'accès sera facilité aux classes préparatoires aux grandes écoles, réservé pour $5 \%$ des plus méritants des lycées d'enseignement général ou technique. Enfin, un programme de busing est proposé aux communes, pour que certains élèves inscrits dans des établissements dont le taux d'échec scolaire est important puissent être transportés dans d'autres écoles de la même ville et échapper à la pression de leur environnement social et scolaire. Mais jusqu'ici, sept villes seulement ont mis en place un tel projet ! De même, la création d'internats éducatifs est présentée comme une autre réponse pour permettre à des jeunes de s'arracher à l'attraction de la rue et des bandes.

Un troisième volet souligne que la sécurité est un droit y compris dans les quartiers, un droit réclamé par les habitants eux-mêmes. Les Unités territoriales de quartier (distinguées de la police de proximité mise en place un temps par la gauche et jugée trop " sociale ») devraient permettre une présence plus habituelle et moins intrusive des forces de police chargées de réprimer les trafics et les incivilités. Mais ce qui est envisagé, c'est aussi la création de " délégués à la cohésion police-population ", sorte de réservistes de la police nationale, consacrés à l'éducation à la citoyenneté.

Le quatrième axe, plus classique, celui de la rénovation urbaine, appelle une coopération des municipalités, des bailleurs, des copropriétés, pour améliorer le cadre de vie (immeubles et espaces publics), mais avec eux aussi la coopération des acteurs économiques et associatifs pour revitaliser l'animation des quartiers. Le Fonds d'intervention pour les services, l'artisanat et le commerce sera réorienté pour financer la réhabilitation des commerces dans les cités. L'État souhaite professionnaliser les acteurs associatifs, mieux valoriser et évaluer leurs actions, créer avec les communes volontaires des «fonds de participation des habitants " pour soutenir 
les initiatives de proximité. Cette politique urbaine comporte, enfin, un effort de désenclavement, avec une amélioration des transports entre les cités et les lieux de travail. Mais ici, les projets (tramway jusqu’à ClichyMontfermeil par exemple) voient toujours leurs échéances retardées.

Ce plan est mis en route au moment même où sont relancées les opérations financées par l'Agence nationale de rénovation urbaine (Anru), qui se traduisent par de lourdes opérations de démolition-reconstruction. Celles-ci, qui ont la faveur des élus locaux en raison des compléments financiers apportés à leur programme de réhabilitation, mettent davantage l'accent sur le bâti : le social y est souvent le parent pauvre.

\section{Limites et résistances}

Est-ce là finalement le plan Marshall pour les banlieues annoncé par Nicolas Sarkozy? Il l'est plus dans les ambitions et le changement de perspectives que par les moyens. Certes, la volonté n'est pas de continuer d'engloutir toujours plus dans des dispositifs réparateurs, dont on dénonce les échecs. Les financements prévus viennent, pour une grande part, du redéploiement des crédits d'autres programmes (déjà prévus pour le Grenelle de l'environnement, la politique de l'emploi...). Mais la politique de la ville risque de s'arrêter à une addition de programmes juxtaposés. Le rêve, l'espoir, de Fadela Amara est de casser cette logique. Il n'est pas évident que le plan y parvienne. La présence de 350 délégués des Préfets dans les quartiers, qui devaient figurer la vigilance de l'État pour ce décloisonnement, se met difficilement en place. Fin décembre 2008, une quinzaine seulement étaient nommés. Un chiffre scandaleux aux yeux de Fadela Amara, qui dénonçait l'inertie de l'administration. Aujourd'hui, 100 délégués sont à leur poste. Les difficultés de la réorganisation des services publics, dans un contexte de révision générale - en vue d'une rationalisation et d'une plus grande efficacité -, ne favorisent pas une présence de proximité, hormis celle de la police. Un vrai plan interministériel ? On peut en douter.

Surtout, il n'est pas sûr que tous les acteurs de la politique de la ville soient invités à contribuer ensemble à un diagnostic local. On leur demande une participation dans des domaines particuliers plus qu'on ne voit en eux un levier pour une mobilisation plus large de toute la société afin d'améliorer le vivre ensemble et lutter contre les ségrégations sociales et spatiales. En ce sens, l'évaluation qui sera faite cette année des Contrats urbains de cohésion sociale (Cucs) avant leur renouvellement, antérieurs au plan Banlieue, sera un rendez-vous important.

Bertrand Cassaigne 